DRAFT VERSION NOVEMBER 20, 2018

Preprint typeset using LTE $_{\mathrm{E}} \mathrm{X}$ style emulateapj v. 11/26/04

\title{
THE MASS-LOSS INDUCED ECCENTRIC KOZAI MECHANISM: A NEW CHANNEL FOR THE PRODUCTION OF CLOSE COMPACT OBJECT-STELLAR BINARIES.
}

\author{
Benjamin J. ShappeE ${ }^{1}$ And TOdD A. ThOMPSON ${ }^{2}$ \\ Department of Astronomy, The Ohio State University, Columbus, Ohio 43210, USA \\ Draft version November 20, 2018
}

\begin{abstract}
Over a broad range of initial inclinations and eccentricities an appreciable fraction of hierarchical triple star systems with similar masses are essentially unaffected by the Kozai-Lidov mechanism (KM) until the primary in the central binary evolves into a compact object. Once it does, it may be much less massive than the other components in the ternary, enabling the "eccentric Kozai mechanism (EKM):" the mutual inclination between the inner and outer binary can flip signs driving the inner binary to very high eccentricity, leading to a close binary or collision. We demonstrate this "Mass-loss Induced Eccentric Kozai" (MIEK) mechanism by considering an example system and defining an ad-hoc minimal separation between the inner two members at which tidal affects become important. For fixed initial masses and semi-major axes, but uniform distributions of eccentricity and cosine of the mutual inclination, $\sim 10 \%$ of systems interact tidally or collide while the primary is on the MS due to the KM or EKM. Those affected by the EKM are not captured by earlier quadrupole-order secular calculations. We show that fully $\sim 30 \%$ of systems interact tidally or collide for the first time as the primary swells to AU scales, mostly as a result of the KM. Finally, $\sim 2 \%$ of systems interact tidally or collide for the first time after the primary sheds most of its mass and becomes a WD, mostly as a result of the MIEK mechanism. These findings motivate a more detailed study of mass-loss in triple systems and the formation of close NS/WD-MS and NS/WD-NS/WD binaries without an initial common envelope phase.
\end{abstract}

Subject headings: stars: binaries: close, — celestial mechanics, stellar dynamics — stars:white dwarfs — supernovae: general

\section{INTRODUCTION}

Kozai (1962) and Lidov (1962) showed that a hierarchical triple system with an inner binary of masses $m_{0}$ and $m_{1}$, and a tertiary of mass $m_{2}$, can exchange angular momentum between the inner and outer orbits periodically. These oscillations drive the eccentricity of the inner binary $\left(e_{1}\right)$ to high values if the tertiary is inclined with $39.2^{\circ} \lesssim i \lesssim 141.8^{\circ}$. For nearly circular orbits, in the test-particle approximation $\left(m_{1} \ll m_{0}, m_{2}\right)$, the maximum eccentricity is given by (Innanen et al. 1997)

$$
e_{\mathrm{in}, \max }=\left(1-\frac{5}{3} \cos ^{2} \mathrm{i}\right)^{1 / 2},
$$

when the three-body Hamiltonian is expanded to quadrupole order in the ratio of the inner to outer semi-major axes $\left(a_{1} / a_{2}\right)$, and neglecting tidal forces and general relativity (Blaes et al. 2002; Miller \& Hamilton 2002; Fabrycky \& Tremaine 2007).

Tidal friction in Kozai-affected triples can cause $a_{1}$ to decrease. As the inner binary is driven to high eccentricity, the periastron of the secondary approaches the primary, and tidal friction tends to circularize the orbit (Mazeh \& Shaham 1979). This mechanism has been proposed as an explanation for the prevalence of "hot" Jupiters with few-day orbits around their host stars (Wu \& Murray 2003; Fabrycky \& Tremaine 2007; Wu et al. 2007; Naoz et al. 2011a b), the very high triple fraction of close solar-type binaries with periods less than $\sim 5$ days (Tokovinin et al. 2006; Fabrycky \& Tremaine 2007), and the formation of blue stragglers in globular clusters (Perets \& Fabrycky 2009). The

Electronic address: shappee@astronomy.ohio-state.edu, thompson@astronomy.ohio

${ }^{1}$ NSF Graduate Fellow

${ }^{2}$ Center for Cosmology \& Astro-Particle Physics, The Ohio State University, Columbus, Ohio 43210, USA combination of Kozai cycles and tidal friction has also been explored generally for triple star systems in Kiseleva et al. (1998) and Eggleton \& Kiseleva-Eggleton (2001). As discussed by Wu \& Murray (2003), for the case of Jupiter-mass planets around Sun-like stars, by equating the tidal and Kozai forcing terms in the equation for the secular evolution of $e_{1}$, one estimates the circularization semi-major axis of the inner binary to be $\sim 3 \mathrm{R}_{\odot}$.

Lithwick \& Naoz (2011), Katz et al. (2011), and Naoz et al. (2011a.b) have recently emphasized that the commonly used quadrupole-order expansion of the threebody Hamiltonian is insufficient to capture the secular dynamics of triple systems in the test particle approximation when the outer eccentricity $\left(e_{2}\right)$ is non-zero. The parameter

$$
\epsilon_{\mathrm{oct}}=\left(\frac{m_{0}-m_{1}}{m_{0}+m_{1}}\right)\left(\frac{a_{1}}{a_{2}}\right) \frac{e_{2}}{1-e_{2}^{2}}
$$

measures the importance of the octupole-order terms in the doubly-averaged three-body Hamiltonian relative to the quadrupole-order terms. Naoz et al. (2011a) first showed that when $\left(m_{1} \ll m_{0}, m_{2}\right)$ and $e_{2} \neq 0$, it is possible for the triple to "flip": the system exhibits quasi-periodic cycles in cos $i$ through 0 , and the tertiary passes from prograde to retrograde and vice versa. These flips occur even for $\epsilon_{\text {oct }}$ as small as $10^{-3}$ in the test particle approximation, provided that the system is sufficiently inclined initially and the arguments of periastron of the inner orbit $\left(g_{1}\right)$ and the outer orbit $\left(g_{2}\right)$ are chosen judiciously (see Figures 7 and 3 of Lithwick \& NaOz 2011 and Katz et al. 2011, respectively). During these flips, the inner binary is driven to extremely high eccentricities $\left(1-\rho_{1} \sim 10^{-5}\right)$. This behavior occurs for a broad range of parandeters in octupole-order calculations, but it is not present in the quadrupole-order calculations, and has been referred to as the "eccentric Kozai mechanism." 
An important piece of physics missing from the test particle approximation of the eccentric Kozai mechanism is the $\left(m_{0}-\right.$ $m_{1}$ ) term in $\epsilon_{\text {oct }}$ (Krymolowski \& Mazeh 1999; Ford et al. 2000; Blaes et al. 2002; Naoz et al. 2011b). Because the three-body Hamiltonian in Jacobi coordinates, expanded to all orders in $a_{2} / a_{1}$ has a sum over $\left(m_{0}^{j-1}-\left(-m_{1}\right)^{j-1}\right)$ for $j=$ $2,3, \cdots$, when $m_{0}=m_{1}$ all $j=$ odd terms are zero (Harrington 1968). Importantly, the flip phenomenon present in the testparticle calculations at modest $e_{2}$ and $i$ is strongly suppressed as $m_{0} \rightarrow m_{1}$ because the octupole-order terms become negligible $^{3}$. Our own experiments with the octupole-order code based on Blaes et al. (2002) and Thompson (2011) shows that in order for flips to occur over a broad range in $g_{1}$ and $g_{2}, m_{1}$ must be less than $m_{0}$ by a factor of $\sim 2$ for a given $a_{1} / a_{2}$ and $e_{2}$.

The dependence of the flip phenomenon on $\left(m_{0}-m_{1}\right)$ implies that many systems will be affected by mass-loss as the primary becomes a WD or a NS. Indeed, many MS triple star systems will have $m_{0} \sim m_{1}$, because the inner binaries of solar-like triples typically have a flat mass distribution (Mazeh et al. 1992; Raghavan et al. 2010) and may even have a preference for "twins" (Pinsonneault \& Stanek 2006; Raghavan et al. 2010). The similar masses of the inner binary suppresses the eccentric Kozai mechanism since $\epsilon_{\text {oct }}$ is small.

Furthermore, a significant fraction of triple systems (those with modest mutual inclination) will have inner binaries that are wide enough to avoid tidal contact and collisions on the MS, even if the system undergoes normal Kozai-Lidov oscillations with $e_{\max }$ given approximately by Equation (1). However, once the primary of the inner binary evolves off the MS and loses the majority of its mass, the system effectively enters the test-particle approximation in many cases, particularly for intermediate- and high-mass primaries where the change in mass as the MS star becomes a compact object is large. After mass loss, $\epsilon_{\text {oct }}$ can increase dramatically, allowing the systems to exhibit the eccentric Kozai mechanism, and driving the inner binary to extremely high eccentricities and tidal contact or collision. The likely result of the MIEK mechanism is a close compact-object(WD/NS)-MS binary with separation $\sim \mathrm{R}_{\odot}$. Such a configuration would produce a variety interesting astrophysical systems.

In this paper we demonstrate this "Mass-Loss Induced Eccentric Kozai" (MIEK) mechanism and provide a preliminary exploration of its dependence on the initial eccentricities and mutual inclination of the triple system, saving a detailed treatment for a future paper. In Section 2, we describe our method for integrating the orbits of triple systems with mass-loss, and we give two example systems with different mass-loss timescales to illustrate the MIEK mechanism. By directly integrating triple systems with an $N$-body code we circumvent the need to pick a limiting order when expanding the threebody Hamiltonian and solving the secular dynamics. In Section 4 we explore parameter space by investigating the effects of eccentricity and inclination on the MIEK mechanism for an example ternary. We find that a fraction of widely separated triple systems are brought to tidal contact for the first time only after the primary evolves off the MS. In Section 5. we discuss the possible outcomes and the implications of systems whose inner binary comes to tidal contact or collides

\footnotetext{
${ }^{3}$ Intuitively, any odd-order terms in the multipole expansion of the threebody Hamiltonian are odd functions of $r_{01}$, the separation vector between members of the inner binary, and thus averages to zero for symmetric inner masses.
}

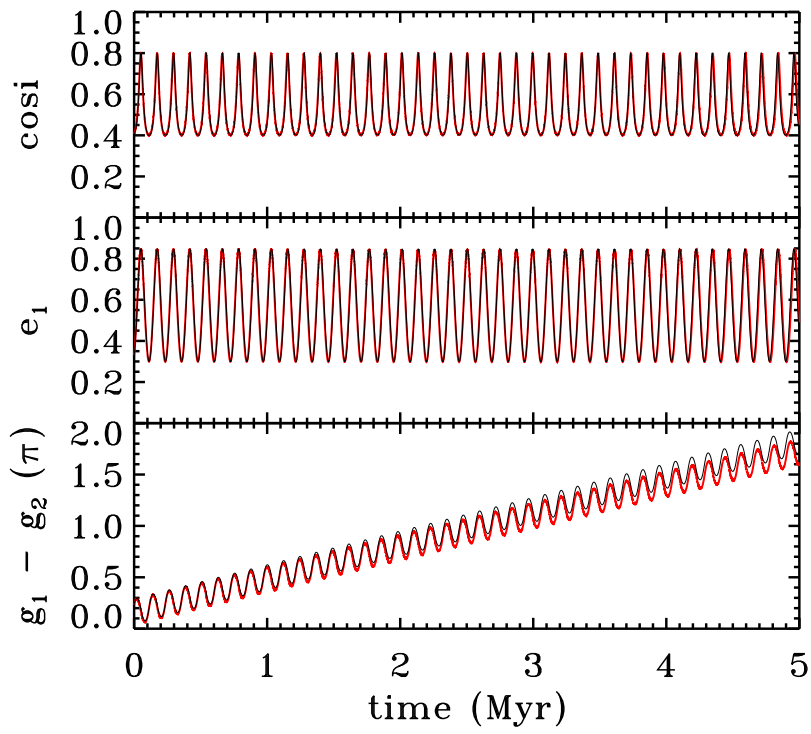

FIG. 1.-Comparison between our modified FEWBODY code (black), and an octupole-order secular code (red,Blaes et al. 2002). The triple system has $m_{0}=7.0 \mathrm{M}_{\odot}, m_{1}=6.5 \mathrm{M}_{\odot}, m_{2}=6 \mathrm{M}_{\odot}, a_{1}=10 \mathrm{AU}, a_{2}=250 \mathrm{AU}, e_{1}=0.3$, $e_{2}=0.1, g_{1}=0^{\circ}, g_{2}=0^{\circ}$, and $\cos i=0.4$. There is a good agreement between the calculations but there is a slow drift in $g_{1}-g_{2}$. Figure discussed in $\$ 2$

at each evolutionary phase. In Section 6, we provide a brief conclusion.

\section{SIMULATING MASS-LOSS IN TRIPLES}

To simulate the dynamical effects of mass-loss on hierarchical triple systems, we modified the $N$-body code FEWBODY ${ }^{4}$ (Fregeau et al.2004) to create triple systems and evolve them. We create a triples with specified inner and outer semi-major axess $\left(a_{1}\right.$ and $\left.a_{2}\right)$, eccentricities $\left(e_{1}\right.$ and $\left.e_{2}\right)$, arguments of periastron $\left(g_{1}\right.$ and $\left.g_{2}\right)$, primary mass in the central binary $\left(m_{0}\right)$, secondary mass in the central binary $\left(m_{1}\right)$, tertiary mass $\left(m_{2}\right)$, random phase angles, and mutual inclination between the two orbits $(\cos i)$.

During integration of the triple, we check that the triple remains bound and stable. If the triple becomes unbound we continue integrating the system until a conservative minimum tidal perturbation on the binary is met $\left(\delta<10^{-7}\right.$; see Fregeau et al. 2004), and we thus include any possible resonant interactions which could result when the triple system becomes unstable ${ }^{5}$. Integration is also stopped if the time steps become exceedingly small. This occurs for a handful of triples where the separation between the inner binary approaches 0 because the stars are on nearly radial orbits. However, in the current study these cases only occur when the inner two stars are separated by $\ll \mathrm{R}_{\odot}$.

To check our modified FEWBODY integrator we tested a sample of triple systems against the N-body integrator MERCURY (Chambers \& Migliorini 1997) and found good agreement. We then tested our code against the octupole-order code of Blaes et al. (2002) with the general relativistic terms removed. An example triple system is shown in Figure 1. As can be seen in the figure there is reasonable agreement with the secular octupole-order calculation. There is, however, a slow drift in $g_{1}-g_{2}$ between the N-body codes and the secular code.

\footnotetext{
${ }^{4}$ FEWBODY is now available at http://fewbody.sourceforge.net/

5 This allows our calculations to include systems affected by the "triple evolution dynamical instability" (TEDI, Perets \& Kratter 2012).
} 


\subsection{Evolutionary Phases}

When we evolve a triple system, we break its evolutionary phases into four distinct stages: (1) primary on the MS, (2) primary evolved into a giant but prior to significant mass loss, (3) during a period of significant mass loss, and (4) after the end of mass loss when the primary has become a compact object. However, because of the prohibitive computational time required to integrate close and highly eccentric triples for the full MS lifetime of the primary, we truncate our integrations after $10^{3}$ Kozai-Lidov times $\left(t_{\mathrm{K}}\right)$ defined by (Innanen et al. 1997; Holman et al. 1997):

$$
\begin{aligned}
t_{\mathrm{K}} & =\frac{4}{3}\left(\frac{a_{1}^{3}\left(m_{0}+m_{1}\right)}{G m_{2}^{2}}\right)^{1 / 2}\left(\frac{b_{2}}{a_{1}}\right)^{3} \\
& \simeq 6.4 \times 10^{4} \mathrm{yr}\left(\frac{a_{1}}{10 \mathrm{AU}}\right)^{3 / 2}\left(\frac{m_{0}+m_{1}}{13.5 \mathrm{M}_{\odot}}\right)^{1 / 2}\left(\frac{6 \mathrm{M}_{\odot}}{m_{2}}\right)\left(\frac{1}{25} \frac{b_{2}}{a_{1}}\right)^{3}
\end{aligned}
$$

where $b_{2}=a_{2}\left(1-e_{2}\right)^{1 / 2}$. Thus, we implicitly assume that the triple systems have no interesting behavior after this time.

We account for the MS lifetime of the primary by only integrating the triple systems for a time $\left(t_{\mathrm{MS}}\right)$ defined by:

$$
t_{\mathrm{MS}}=\min \left(t_{\mathrm{ms}, 0}, 10^{3} t_{\mathrm{K}}\right) \times(0.95+0.1 \xi),
$$

where $\xi$ is a uniformly distributed random number on the interval $[0,1), t_{\mathrm{ms}, 0}$ is the MS lifetime of the primary. We determine the MS lifetimes by interpolating the logarithmic lifetimes on a grid of stellar models run with the Yale Rotating Evolution Code with input physics described in van Saders \& Pinsonneault (2011). We multiply by the second factor in Equation (4) in order to randomize exactly when the primary evolves off the main sequence to avoid any correlations of the start of mass loss between different triple systems in our sample.

To simulate the giant phase of the primary, we continue to integrate the triple for a time $\left(t_{\mathrm{RG}}\right)$ defined by:

$$
t_{\mathrm{RG}}=\min \left(0.1 t_{\mathrm{ms}, 0}, 10^{3} t_{\mathrm{K}}\right) .
$$

Then, to simulate mass-loss, we linearly decrease $m_{0}$ over a mass-loss time scale $\left(t_{\mathrm{ml}}\right)$ to the WD mass as specified by the initial-final mass relation of Kalirai et al. (2008) given by:

$$
m_{0, \mathrm{f}}=(0.109 \pm 0.007) m_{0}+(0.394 \pm 0.025) \mathrm{M}_{\odot},
$$

where $m_{0, \mathrm{f}}$ is final mass of the primary. To test our mass-loss prescription, we integrated binaries where one member underwent mass loss and tested that the change in the semi-major axis and eccentricity of the binary matched that for instantaneous mass loss and adiabatic mass loss (i.e., Equations 4-6 in Baribault \& Pineault 1994) when $t_{\mathrm{ml}} \ll P$ and when $t_{\mathrm{ml}} \gg P$, respectively, where $P$ is the orbital period of the orbit. We found good agreement.

After mass-loss has finished we integrate the evolved triple for a time $\left(t_{\text {after }}\right)$ given by:

$$
t_{\mathrm{after}}=\min \left(t_{\mathrm{ms}, 1}-1.1 t_{\mathrm{ms}, 0}-t_{\mathrm{ml}}, 10^{3} t_{\mathrm{K}}\right),
$$

where $t_{\mathrm{ms}, 1}$ is the MS lifetime of the next most massive star in the triple system and $t_{\mathrm{K}}$ is redefined by Equation (3) with the orbital parameters of the triple right after the conclusion of mass-loss.

\subsection{Tidal Contact and/or Collisions}

A detailed treatment of stellar tides and tidal dissipation is beyond the scope of this paper. However, to determine when tidal effects are likely to be important, we define an ad-hoc minimal separation between the inner two binary members, $r_{\text {tide }}$, below which strong tidal contact or a collision is assumed to have occurred. That is, if the separation between the primary and secondary is $r<r_{\text {tide }}$, we assume either (1) that tidal dissipation will remove energy from the inner orbit decreasing $a_{1}$ or (2) if the separation between the inner binary members changes dramatically in a single inner orbital period $\left(P_{1}\right)$, a physical collision may occur before tides become important, as in the recent work of Katz \& Dong (2012). In either case, our simulations are not valid for $r<r_{\text {tide }}$.

When choosing a tidal criterion we considered two tidal effects which will alter the evolution of the system. The first is the nondissipative contribution that tidal bulges on the members of the inner binary have on apsidal motion (Sterne 1939). This additional apsidal motion may detune the Kozai mechanism (e.g. Wu \& Murray 2003; Fabrycky \& Tremaine 2007). We find that the timescale of this apsidal motion becomes comparable to $t_{\mathrm{K}}$ when $r_{\text {peri }} \sim 4 R_{\mathrm{ms}, 0}$, assuming the typical tidal Love number valid for $n=3$ polytropes, $k=0.028$, (Eggleton \& Kiseleva-Eggleton 2001) and the system parameters for the fiducial system presented in $\$ 3$. The second effect is eccentricity damping due to dissipative tides (Mazeh \& Shaham 1979; Wu et al. 2007). For our fiducial system, we derive a critical inner seperation by equating the tidal and Kozai forcing terms in the equation for the secular evolution of $e_{1}$. We find a critial inner separation of:

$$
r_{\text {crit }} \sim 3 R_{\mathrm{ms}, 0}\left(\frac{10^{4}}{Q} \frac{k}{0.028}\right)^{2 / 13},
$$

where $Q$ is the tidal dissipation factor. As can be seen in Equation (8), $r_{\text {crit }}$ is very insensitive to the exact values chosen for $k$ and $Q$. For simplicity, and to allow a broad, albeit incomplete, survey of parameter space, we take $r_{\text {tide }}=$ $3 \times \max \left(R_{m s, 0}, R_{m s, 1}\right)$ for the bulk of this study, but we investigate the effect of changing $r_{\text {tide }}$ on our results in $\$ 4.3$

\subsection{General Relativistic Precession}

General relativistic (GR) periastron precession can "detune" the normal Kozai mechanism, and truncate the maximum eccentricity attainable for a triple system (Blaes et al. 2002; Miller \& Hamilton 2002; Thompson 2011). FEWBODY does not contain post-Newtonian corrections, so we must check if it is permissible to ignore GR precession. The GR precession timescale (e.g., Equation 23 of Fabrycky \& Tremaine 2007) is given by:

$$
\begin{aligned}
t_{\mathrm{GRp}} & =\frac{1}{3} \frac{a_{1}}{c}\left(\frac{a_{1} c^{2}}{G\left(m_{0}+m_{1}\right)}\right)^{3 / 2}\left(1-e_{1}^{2}\right) \\
& \simeq 3.4 \times 10^{7} \mathrm{yr}\left(\frac{13.5 \mathrm{M}_{\odot}}{\left(m_{0}+m_{1}\right)}\right)^{3 / 2}\left(\frac{a_{1}}{10 \mathrm{AU}}\right)^{5 / 2}\left(1-e_{1}^{2}\right) .
\end{aligned}
$$

As discussed in Blaes et al. (2002), the Kozai mechanism only operates if $t_{\mathrm{K}} \lesssim t_{\mathrm{GRp}}$. We see that for the masses and semimajor axes of our fiducial case presented in $\$ 3, t_{\mathrm{K}} \lesssim t_{\mathrm{GRp}}$ for any choice of $e_{2}$ until the inner eccentricity is increased such that $r_{\text {peri }}<R_{\mathrm{ms}, 1}$. Thus the normal Kozai mechanism is free to operate in our triple integrations.

However, we also see in $\$ 3$ that the eccentric Kozai timescale $\left(t_{\mathrm{EK}}\right)$, the timescale for "flips" and extreme eccentricity maxima, is much longer then $t_{\mathrm{K}}, t_{\mathrm{EK}} \sim 10^{6}$ years. 
Furthermore, we see that $t_{\mathrm{EK}}>t_{\mathrm{GRp}}$ for values of $e_{1}$ where $r_{\text {peri }}>\mathrm{R}_{\odot}$, which would seem to imply that GR precession might detune the eccentric Kozai mechanism before tidal effects become important. This is not clear however, because the majority of the GR precession occurs when $e_{1}$ is large, which is only a small fraction of the eccentric Kozai cycle. To test if GR precession will suppress the maximum $e_{1}$ reached, we take the fiducial triple system after mass loss that exhibits the eccentric Kozai flip, and compare this to the equivalent octupole-order calculation that includes GR precession using the code of Thompson (2011) (Blaes et al. 2002). We found good agreement between the two calculations, implying that GR precession does not shut off the eccentric Kozai mechanism, and is thus not important for the current study. However, these effects should be more carefully considered in future studies where $a_{1}$ is much smaller, and when the periastron distance can be significantly smaller than $R_{\odot}$ if both the primary and secondary are compact objects.

\section{MIEK:}

\section{THE MASS-LOSS INDUCED ECCENTRIC KOZAI MECHANISM}

Figure 2 presents two example systems with the left and right panels having mass-loss time scales of $t_{\mathrm{ml}}=10^{4} \mathrm{yr}$ and $t_{\mathrm{ml}}=10^{6} \mathrm{yr}$, respectively. Both have $m_{0}=7.0 \mathrm{M}_{\odot}, m_{1}=$ $6.5 \mathrm{M}_{\odot}, m_{2}=6 \mathrm{M}_{\odot}, a_{1}=10 \mathrm{AU}, a_{2}=250 \mathrm{AU}, e_{1}=0.1$, $e_{2}=0.7, g_{1}=0^{\circ}, g_{2}=180^{\circ}$, and $\cos i=0.5\left(i=60^{\circ}\right)$. The top panels present the evolution of $a_{1}, a_{2}, e_{2}$, and $m_{0}$. The middle and bottom panels show the evolution of $\cos i$ and $1-e_{1}$, respectively. In the bottom panel, the red line shows where $e_{1}$ is high enough such that the radius of periastron of the inner binary $\left(r_{\text {peri }}\right)$ is equal to $\mathrm{R}_{\odot}$. The example systems go through normal Kozai-Lidov cycles before mass-loss begins, reaching a maximum $e_{1} \sim 0.75$ as predicted by Equation (1), but $r_{\text {peri }}$ is still more than two orders of magnitude too large for tidal interactions to be significant.

After adiabatic mass-loss $m_{0} \rightarrow 1.15 \mathrm{M}_{\odot}$, and $\epsilon_{\text {oct }}$ increases (Equation 2). To calculate this increase analytically, we first note that the increase in the semi-major axis and change in eccentricity of a binary that underwent adiabatic mass-loss is given by:

$$
\frac{a_{\mathrm{f}}}{a_{0}}=\frac{M_{0}}{M_{\mathrm{f}}}, \text { and } e_{\mathrm{f}}=e_{0},
$$

where $M_{0}$ and $M_{\mathrm{f}}$ are the initial total mass and final total mass of the binary, respectively. From Equation (10) we see that the change in the ratio of the semi-axes of a triple system that underwent adiabatic mass-loss is given by:

$$
\frac{a_{2, \mathrm{f}}}{a_{1, \mathrm{f}}}=\frac{a_{2, \mathrm{i}}}{a_{1, \mathrm{i}}}\left(\frac{m_{0}+m_{1}+m_{2}}{m_{0, \mathrm{f}}+m_{1}+m_{2}}\right)\left(\frac{m_{0, \mathrm{f}}+m_{1}}{m_{0}+m_{1}}\right),
$$

where $a_{2, \mathrm{i}}$ and $a_{2, \mathrm{f}}$ are the initial and final outer semi-major axes, respectively, and $a_{2, \mathrm{i}}$ and $a_{2, \mathrm{f}}$ are the initial and final inner semi-major axes, respectively. From Equation (11) one sees that the outer to inner semi-major axis ratio decreases for our fiducial system during adiabatic mass loss by $a_{2, \mathrm{f}} / a_{1, \mathrm{f}} \simeq$ $0.8 a_{2, \mathrm{i}} / a_{1, \mathrm{i}}$. Then with Equation (2) we see that the fractional increase ${ }^{6}$ in $\epsilon_{\text {oct }}$ due to adiabatic mass loss of the primary is given by:

$$
\frac{\epsilon_{\mathrm{oct}, \mathrm{f}}}{\epsilon_{\mathrm{oct}, \mathrm{i}}}=\left|\left(\frac{m_{0, \mathrm{f}}-m_{1}}{m_{0}-m_{1}}\right)\left(\frac{m_{0, \mathrm{f}}+m_{1}+m_{2}}{m_{0}+m_{1}+m_{2}}\right)\left(\frac{m_{0}+m_{1}}{m_{0, \mathrm{f}}+m_{1}}\right)^{2}\right|,
$$

${ }^{6}$ While $\epsilon_{\mathrm{oct}, \mathrm{i}}$ and $\epsilon_{\mathrm{oct}, \mathrm{f}}$ will vary between systems in 8 depending on $e_{2}$, the fractional increase of $\epsilon_{\mathrm{oct}}$ will, however, be the same because the mass loss is adiabatic so $e_{2}$ drops out. where $\epsilon_{\mathrm{oct}, \mathrm{i}}$ and $\epsilon_{\mathrm{oct}, \mathrm{f}}$ are the initial and final values of $\epsilon_{\mathrm{oct}}$, respectively. The absolute value is due to the ambiguity as to which of the binary stars will be the most massive in the final system. After mass loss our fiducial triple system is in the near-test-particle limit and $\epsilon_{\text {oct }}$ increases by a factor of 23.3, from 0.002 to 0.047 . These factors enable the eccentric Kozai mechanism, the system then flips, and as cos $i$ passes through $0,\left(1-e_{1}\right)$ approaches $10^{-4}$. Both systems would be affected by tidal interactions during their first flip as $r_{\text {peri }}<\mathrm{R}_{\odot}$. This example illustrates the essence of the MIEK mechanism.

\section{INCLINATION AND ECCENTRICITY DEPENDENCE}

The importance of the MIEK mechanism for a full population of triple systems will depend on the adopted mass function of the stars, the eccentricity and semi-major axis distributions of the inner binary and outer tertiary, as well as the mutual inclination distribution. We save a full exploration of parameter space for a future paper, but we explore the effects of inclination and eccentricity on the dynamics of the example system from \$3. This system is an interesting test case because all the masses are approximately equal, so the octupoleorder terms mostly become important during and after mass loss.

\subsection{Parameters Space Exploration}

To explore the effects of inclination and eccentricity, we integrate $\sim 10^{5}$ triple systems over an equally spaced grid with $41,10,20$, and 4 values of $\cos i, e_{1}, e_{2}$, and $g_{1}$, respectively, while keeping the initial masses and initial semi-major axes from our example system. Because the outcomes of triple systems effected by MIEK may differ depending on the initial phase angles, we integrate three triple systems at each grid point in $\cos i, e_{1}, e_{2}$, and $g_{1}$ with randomized initial phase angles.

In order to determine if the inner binary of each system becomes tidally affected or collides by our ad-hoc tidal criterion (Section 2.2), we record the minimum inner separation $\left(r_{\min }\right)$ in each of the four evolutionary phases described in $\$ 2$. We integrate these triple systems assuming $t_{\mathrm{ml}}=10^{4}$ years and posit that tides will affect the inner binary if $r_{\min }$ is smaller than some ad-hoc tidal radius $\left(r_{\text {tide }}\right)$. We define this ad-hoc tidal radius differently for the different evolutionary phases of the primary, in an attempt to capture the different strength of tidal forces due to the varying physical size and mass of the primary. Before the primary evolves off the MS we take $r_{\text {tide }}=3 R_{\mathrm{ms}, 0}$, where $R_{\mathrm{ms}, 0}$ in the MS radii of the primary. When the primary is a giant and during the massloss phase, we take $r_{\text {tide }}=1 \mathrm{AU}$. Finally, when the primary becomes a WD we take $r_{\text {tide }}=3 R_{\mathrm{ms}, 1}$, where $R_{\mathrm{ms}, 1}$ in the MS radii of the secondary in the inner binary. We determine $R_{\mathrm{ms}, 0}$ and $R_{\mathrm{ms}, 1}$ from the Torres et al. (2010) sample by taking the median of the observed radii for stars with similar masses $\left(\left|\log M-\log M^{\prime}\right|<0.1\right)$.

Additionally, we would like to determine when the eccentric Kozai mechanism is operating. Because orbital flips (i.e., a change of sign in $\cos i$ ) only occur during the eccentric Kozai mechanism, we monitor each triple systems for flips during each of the aforementioned evolutionary phases. We then use the two above criteria to loosely define systems that are strongly affected by the MIEK mechanism, as systems that come into tidal contact and exhibit a flip after the onset of mass loss.

\subsection{Results}



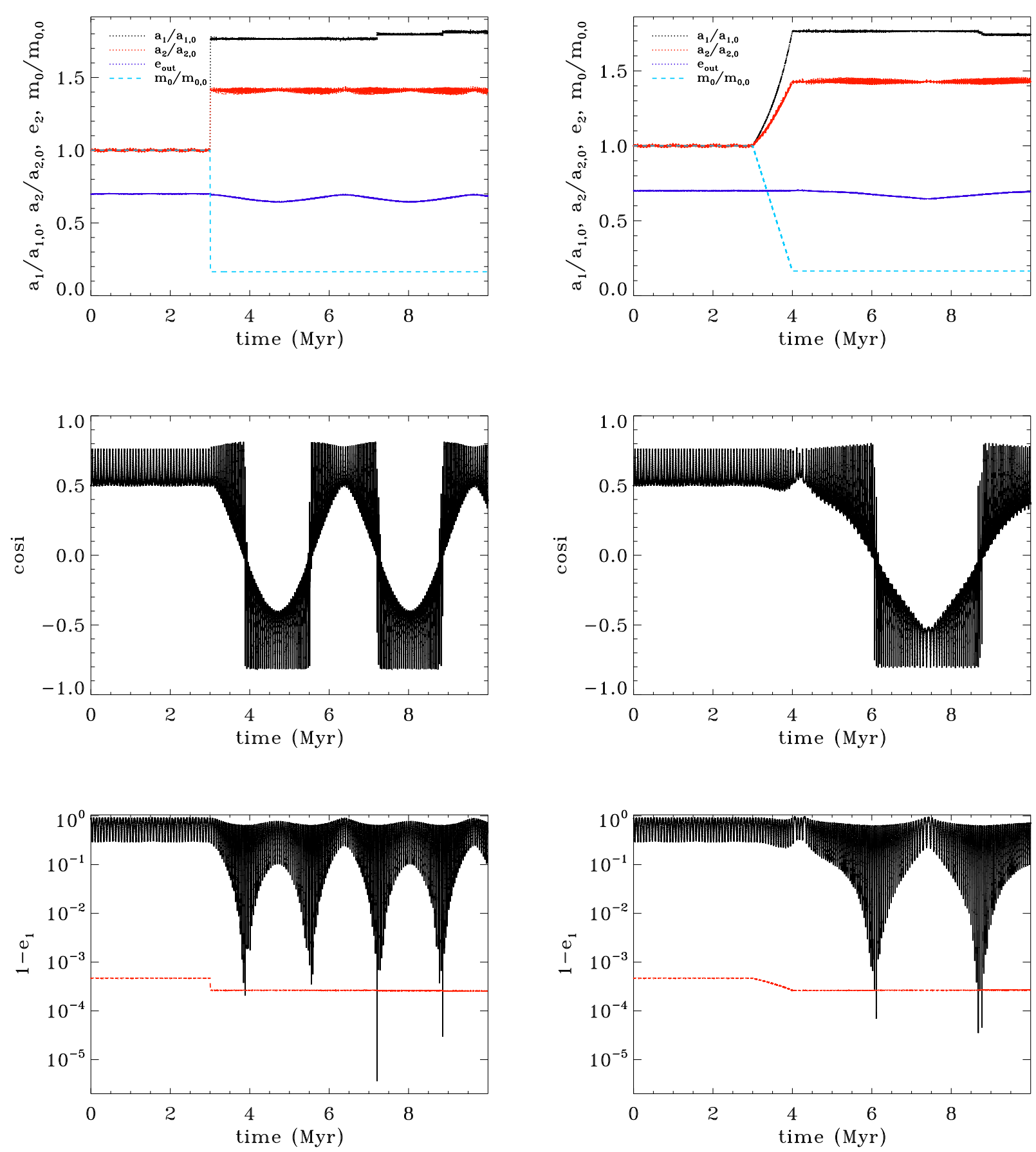

FIG. 2.- The "Mass-loss Induced Eccentric Kozai" (MIEK) Mechanism. Two example systems with the left and right panels having mass-loss timescales of $t_{\mathrm{ml}}=10^{4} \mathrm{yr}$ and $t_{\mathrm{ml}}=10^{6} \mathrm{yr}$, respectively. Triples have $m_{0}=7.0 \mathrm{M}_{\odot}, m_{1}=6.5 \mathrm{M}_{\odot}, m_{2}=6 \mathrm{M}_{\odot}, a_{1}=10 \mathrm{AU}, a_{2}=250 \mathrm{AU}, e_{1}=0.1, e_{2}=0.7, g_{1}=0^{\circ}, g_{2}=180^{\circ}$, and $\cos i=0.5$. Top Panels: Evolution of $a_{1}$ (dotted black), $a_{2}$ (red dotted), $e_{2}$ (blue dotted), and $m_{0}$ (light blue dashed). Middle Panels: Time evolution of cos $i$. Bottom Panels: Time evolution of $e_{1}$. Note the qualitative difference between the normal Kozai-Lidov mechanism $\left(e_{1} \rightarrow 0.7\right)$ before the onset of mass loss at 3 Myr and the eccentric Kozai mechanism $\left(e_{1} \rightarrow 1\right)$ after mass loss when $r_{\text {peri }}<\mathrm{R}_{\odot}$ (denoted by the red dashed line) and flips occur quasi-periodically. We caution that our integrations after the first eccentricity maximum are for purposes of illustration only since we do not include the effects of tides.

The results of the parameter space search in inclination and eccentricity are presented in Table 1. Figure 3, and Figure 4. In Table 1 we break down the fraction of the triple systems that flip and become tidally affected or collide during the various evolutionary stages presented in $\$ 2$. We highlight any asymmetries about $\cos i_{0}=0$ by presenting the fractions for prograde and retrograde systems separately. We now describe each of the evolutionary phases of the primary in turn.
When the primary is on the MS ("Primary MS" in Table 11), $13 \%$ of triple systems become tidally affected or collide $\left(r_{\min } \leq r_{\text {tide }}\right)$. We show the initial inclinations and eccentricities of these triple systems in the left panel of Figure 3. Each contour plot displays the parameter space projected onto a two parameter plane. Each histogram marginalizes over all parameters except one, and then displays the fraction of systems in each bin that become tidally affected or collide. From the left 

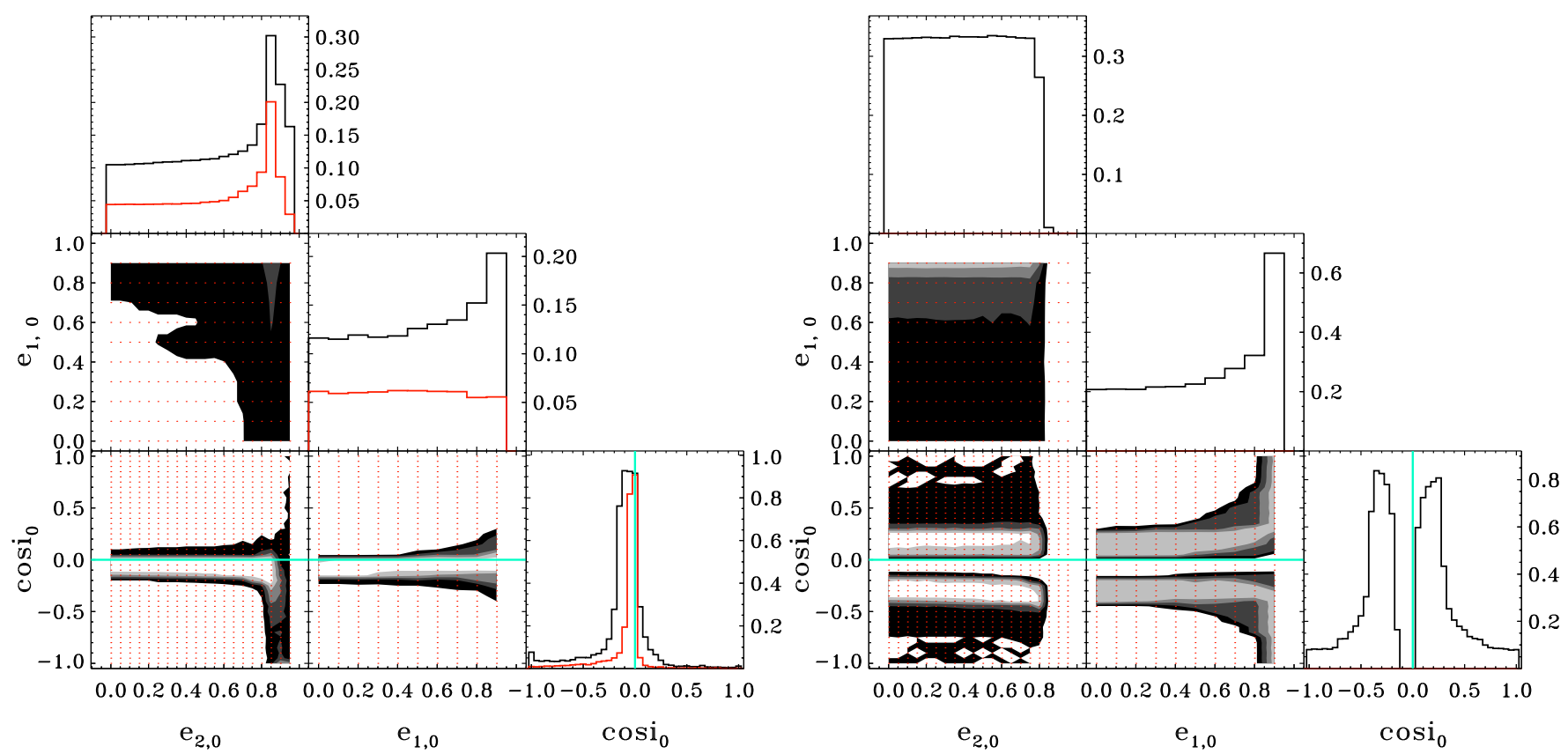

FIG. 3.- Initial parameters for triple systems that were tidally affected or collided on the MS, $r_{\text {peri }}<3 R_{\mathrm{ms}, 0}$ (left), and triple systems that were tidally affected or collided after the primary evolved off the main sequence but before the onset of significant mass-loss, $r_{\text {peri }}<1$ AU (right). In the histograms, black shows the fraction of systems in each bin that are tidally affected or collide and red shows the fraction of systems that also exhibited an orbital flip. Contours present the fraction of systems at each grid point that would be tidally affected by our criteria. The contours are in steps of $20 \%$ starting at $10 \%$ (black) Red dots show the grid points where systems where integrated. The teal line on the contours show where $\cos i=0$. Note that there is a pronounced asymmetry between prograde and retrograde triples. Figure discussed in $\$$

TABLE 1

TRIPLE OUTCOMES

\begin{tabular}{|c|c|c|c|c|}
\hline & Contact & Flip & Contact \& Flip & Unbound \\
\hline \multicolumn{5}{|l|}{ Combined } \\
\hline Primary MS & .13 & 0.064 & 0.060 & 0.11 \\
\hline Primary Giant & 28 & 000041 & 0.000030 & 0.00037 \\
\hline Loss & 0016 & 0.0015 & & 0.0027 \\
\hline After Ma & 02 & & 0.019 & 0.011 \\
\hline \multicolumn{5}{|l|}{ Prograde } \\
\hline Primary MS & 0.039 & 0.0076 & 0.0059 & 0.15 \\
\hline & 30 & 0063 & 0.00 & 0.00042 \\
\hline -Loss & .00013 & 0.0020 & 0.0000 & \\
\hline After Mas & & & 0.02 & \\
\hline \multicolumn{5}{|l|}{ Retrograde } \\
\hline & 22 & 0.12 & 0.11 & 0.08 \\
\hline & 26 & 0020 & 0.0 & 0.00032 \\
\hline Mass-Loss & 0.00020 & 0.00097 & 0.000040 & 0.0017 \\
\hline After Mass-Loss & 0.015 & 0.013 & 0.010 & 0.014 \\
\hline \multicolumn{5}{|c|}{$\begin{array}{l}\text { NOTE. - Fractions of triple systems that become tidally affected, exhibit a flip, or } \\
\text { are unbound at various evolutionary stages without being tidally affected or unbound in } \\
\text { a previous evolutionary stage. Prograde and retrograde divide the sample in half where } \\
\text { combined are the fractions of the whole sample. The rows Primary MS, Primary Giant, } \\
\text { During Mass-Loss and After Mass-Loss present the fractions of triple systems with the } \\
\text { primary star is on the main sequence, a giant that has evolved off the main sequence, } \\
\text { an evolved star undergoing an } 10^{4} \text { year phase of significant mass-loss phase and a WD, } \\
\text { respectively. The columns are as follows. Contact }- \text { Systems that satisfy our ad-hoc } \\
\text { tidal criteria. Flip - Systems whose cos } i \text { changes sign from its initial value. Contact } \\
\& \text { Flip - Systems common to the previous two columns. Unbound - Systems that } \\
\text { become unbound without being tidally affected prior. Table discussed in }\end{array}$} \\
\hline
\end{tabular}

panel of Figure 3, we see that when the primary is on the MS the majority of systems which become tidally affected or collide are initially highly inclined. These systems undergo normal Kozai-Lidov oscillations, which bring the inner binary to tidal contact with a maximum pericenter distance given by:

$$
r_{\text {peri }}=a_{1}\left(1-e_{1, \max }\right)=a_{1}\left[1-\left(1-\frac{5}{3} \cos ^{2} i_{0}\right)^{1 / 2}\right]
$$

with $e_{1, \max }$ given by Equation (1) and $i_{0}$ the initial eccentricity. Then, setting the periastron distance equal to $r_{\text {tide }}$,

$$
r_{\text {peri }}=r_{\text {tide }}=3 R_{\mathrm{ms}, 0} \sim 12 \mathrm{R}_{\odot} \sim 0.05 \mathrm{AU} \sim 0.005 \mathrm{a}_{1},
$$

we see that the required critical initial inclination to interact tidally when the primary is on the MS is

$$
\cos i_{0, \text { crit }} \sim 0.08,
$$

which is in good agreement with the prograde orbits in the left panel of Figure 3 Note, however, that the distribution is asymmetric about $\cos i_{0}=0$, with $22 \%$ of the retrograde systems becoming tidally affected or colliding while only $3.9 \%$ of the prograde systems do. This asymmetry is not captured by Equation (1) and Equation (13), because of the approximations under which Equation (1) is derived. A more complete treatment of the quadrupole-order Hamiltonian shows that $e_{1, \max }$ does not occur at $\cos i_{0}=0$, but instead at retrograde mutual inclination (see Fig. 1 of Miller \& Hamilton 2002).

Importantly, we find that $6.0 \%$ of the triple systems, with $\cos i_{0} \simeq 0$, become tidally affected and exhibit an orbital flip, which is characteristic of the eccentric Kozai mechanism. These systems comprise almost half of the total number of systems that become tidally affected or collide when the primary is on the MS, showing that the eccentric Kozai mechanism is also important. The implications that the eccentric Kozai has for previous studies involving the KozaiLidov mechanism and tidal friction is discussed further in $\$ 5$ but here we note that the systems that tidally interact or collide on the MS are analogous to the systems investigated by Fabrycky \& Tremaine (2007), except that these authors only included quadrupole-order terms in the secular Hamiltonian, and thus may have missed the systems that undergo the eccentric Kozai mechanism and execute a "flip" with $e_{1} \rightarrow 1$ (Naoz et al. 2011a.b).

Additionally, $11 \%$ of triple systems become unbound when the primary is on the MS. The systems that are un- 


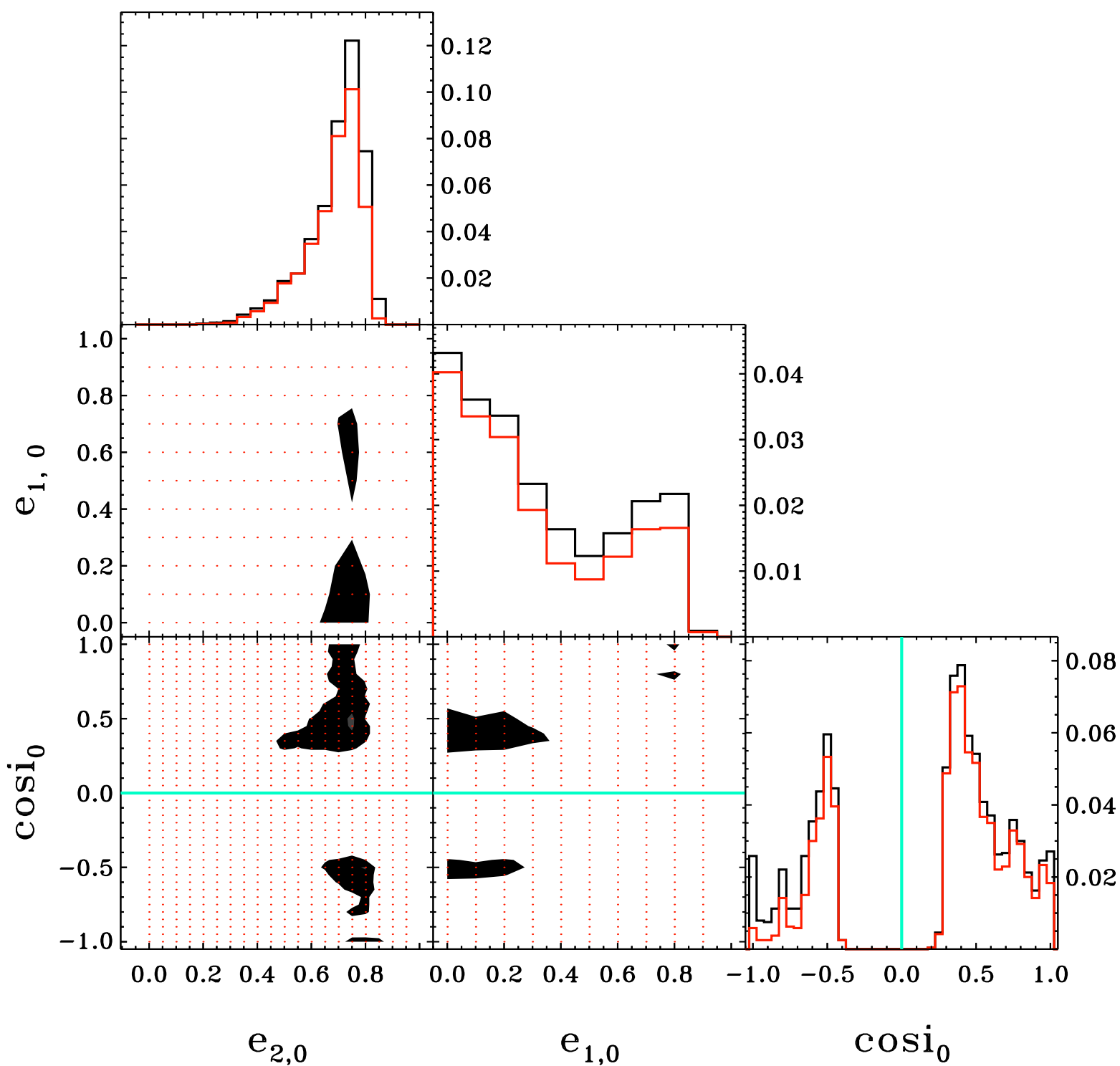

FIG. 4.- Initial parameters for triple systems that were tidally affected or collide after the end of mass loss, $r_{\mathrm{peri}}<3 R_{\mathrm{ms}, 1}$. In the histograms, black shows the fraction of systems in each bin that are tidally affected or collide and red shows the fraction of systems that also exhibited an orbital flip. Contours present the fraction of systems at each grid point that would be tidally affected by our criteria. The contours are in steps of $20 \%$ starting at $10 \%$ (black). Red dots show the grid points where systems where integrated. The teal line on the contours show where cos $i=0$. Note there is a pronounced asymmetry between prograde and retrograde triples. Figure discussed in $\$$

bound have large $e_{2}$ and do not satisfy the standard, empirically derived, stability criterion for triple systems given by (Mardling \& Aarseth 2001):

$$
\frac{a_{2}}{a_{1}} \geq C f\left[\left(1+\frac{m_{2}}{m_{0}+m_{1}}\right) \frac{1+e_{2}}{\left(1-e_{2}\right)^{3}}\right]^{0.4},
$$

where $C=2.8$ and $f=1-\frac{0.3}{\pi} i$ is an ad-hoc inclination term. These systems are present in our study because we uniformly sample the eccentricities without regard for the initial dynamical stability of the system. We see from Equation (16) and our initial assumed semi-major axes, that if $\cos i=0$ then $e_{2} \leq 0.83$ must hold for the system to satisfy the stability criterion. It is interesting to note that in the $\cos i_{0}$ vs. $e_{2,0}$ contour plot in the left panel of Figure 3, many triple systems which are unstable given the stability criteria become tidally affected or collide before they are unbound. It is possible that tidal friction will quickly shrink $a_{1}$ of these systems, causing them to become stable as the ratio $a_{2} / a_{1}$ increases.

After the primary has evolved off the MS but before signif- icant mass-loss has begun ("Primary Giant" in Table 1), 28\% of triple systems become tidally affected or collide $\left(r_{\min } \leq\right.$ $1 \mathrm{AU})$ for the first time. We show the initial inclinations and eccentricities of these triple systems in the right panel of Figure 3 Such a large fraction of the triple systems become tidally affected or collide during this stage of evolution because the physical size of the primary dramatically increases, so we impose an ad-hoc increase to $r_{\text {tide }}=1 \mathrm{AU}$. Then from Equation (13) we find that

$$
\cos i_{0, \text { crit }} \simeq 0.34,
$$

encompassing about a third of our parameter space. Additionally, we see that only a small fraction of the systems exhibit the orbital flip characteristic of the eccentric Kozai mechanism when the primary is a giant, showing that the eccentric Kozai mechanism is not important during this phase of the primary's evolution. Lastly, only $0.037 \%$ of the systems are unbound during this evolutionary phase, emphasizing that initially dynamically unstable systems are either quickly unbound, when the primary is still on the MS, collide, or become 
tidally affected.

During the $t_{\mathrm{ml}}=10^{4}$ year mass-loss phase ("During MassLoss" in Table 1 only a small fraction, $0.016 \%$, of the triple systems become tidally affected or collide for the first time. This is because previous stages of evolution have removed the majority of triple systems that would tidally interact or collide with $r_{\text {tide }}=1 \mathrm{AU}$, and because $t_{\mathrm{ml}}<t_{\mathrm{K}}$, so the systems do not have time to complete a Kozai cycle during the primary's mass loss episode. We find that the fraction of systems which are unbound increases, to $0.27 \%$, from the previous phase of evolution. More systems become unstable because, as shown by Equation (11), the outer to inner semi-major axis ratio decreases during mass loss. Then, as a result of Equation (16), this decrease in the semi-major axis ratio causes more triple systems to be unstable.

Finally, after the end of mass-loss ("After Mass-Loss" in Table 1) we see that $2.2 \%$ of triple systems spanning a broad range in orbital parameters become tidally affected or collide for the first time. We note that the lion's share, $1.9 \%$, of these systems exhibit an orbital flip characteristic of the eccentric Kozai mechanism and thus the MIEK mechanism because it is induced by mass loss of the primary. We now discuss the distribution of tidally affected or colliding systems after mass loss for the inclination and eccentricities individually.

The distribution of these tidally affected or colliding systems is asymmetric about $\cos i_{0}=0$, with $3.0 \%$ of prograde systems and only $1.5 \%$ of retrograde systems becoming tidally affect of colliding. At least some of this asymmetry arises because, like previous stages of evolution of the primary, systems with $\cos i_{0}$ near 0 are strongly preferred. However, earlier stages of evolution have removed a disproportionate fraction of the prograde systems, 33\%, compared to the fraction of retrograde systems, $49 \%$. This causes the reverse asymmetry, more prograde than retrograde systems, in the distribution of systems which become tidally affected or collide after the end of mass loss.

The distribution in $e_{2,0}$ for the systems that become tidally affected or collide after the end of mass loss shows a strong preference for larger values, peaking at $e_{2,0}=0.75$. This preference is easily understood when considering Equation (2), which also shows a strong dependence on $e_{2,0}$. Thus, the octupole order terms and consequently the eccentric Kozai mechanism are expected to become more important with larger $e_{2,0}$. Even though there is a strong preference for large $e_{2,0}$, the MIEK mechanism continues to operate for systems with modest $e_{2,0} \sim 0.4$. We also note that the $e_{2,0}$ distribution is truncated at very large values, which arises because most of these systems became tidally affected, collided, or were unbound when the primary was on the MS.

Lastly, the distribution of $e_{1,0}$ for the systems that become tidally affected or collide shows a preference for both large and small values. The minimum in the distribution occurs at $e_{1,0} \sim 0.5$, with $\sim 3$ times fewer systems becoming tidally affected or colliding then at $e_{1,0}=0$. The preference for large values of $e_{1,0}$ is easily understood because these systems do not need to increase their inner eccentricity as much to collide or come into tidal contact. The preference for smaller values of $e_{1,0}$, however, is not easily understood. We also note that the majority of systems with $e_{1,0}=0.9$ become tidally affected or collide in earlier stages of the primary's evolution, so these systems are absent in the current $e_{1,0}$ distribution.
We now briefly evaluate the sensitivity of the results presented in $\$ 4.2$ on the ad-hoc tidal criterion chosen (Section 2.2).

First, we evaluate the dependence of the fraction of systems that become tidally affected or collide on our choice of tidal criterion. We both decreased and increased the tidal criterion from $r_{\text {tide }}=3 R_{*}$ to $r_{\text {tide }}=2.5 R_{*}, r_{\text {tide }}=3.5 R_{*}, r_{\text {tide }}=5 R_{*}$, and $r_{\text {tide }}=10.0 R_{*} . R_{*}=R_{\mathrm{ms}, 0}$ when the primary is on the MS and $R_{*}=R_{\mathrm{ms}, 1}$ when the primary is a WD. The fraction of systems that become tidally affected or collide after the end of mass-loss with these new tidal criteria are $0.022,0.023$, 0.023 , and 0.025 , respectively. When these fractions are compared to that presented in Table 1, we see that the fraction of systems affected by the MIEK mechanism is relatively insensitive to the exact choice of tidal criterion. This insensitivity results because an increase in the tidal radius on the MS affects systems that will already be affected by the large tidal radius when the primary becomes a Giant, and does not affect regions of parameter space shown in Figure 4 where the MIEK mechanism operates. In fact, increasing the tidal radius marginally increases the fraction of systems affected by MIEK.

Additionally, we emphasize that by eliminating all systems that collide or were tidally affected at a prior stage of evolution, $1.9 \%$ is a conservative estimate of the fraction of triple systems that will undergo the MIEK mechanism. This is because many of the systems we remove from the sample as the evolutionary stages progress may still be viable candidates for the MIEK mechanism. One possibility is that tides on the giant primary only detune the normal Kozai-Lidov mechanism, and do not allow $e_{1}$ to increase so dramatically. Then, our removal of these systems from the sample would unrealistically decrease the number of systems that experience MIEK. These systems would survive in our sample to the stage after mass loss is complete. Another possibility is that the combination of the Kozai-Lidov mechanism and tides on a giant will work to shrink $a_{1}$ to a few AU. These systems will then have $a_{2} / a_{1} \sim 100$, but they can still be affected by the eccentric Kozai mechanism after mass loss, which will shrink this semi-major axis ratio according to Equation (11).

Evaluating these effects would involve a realistic calculation of the 3-body dynamics with tides since $\cos i$ will evolve secularly during the tidal interaction (Fabrycky \& Tremaine 2007). We save this for a future work. Here, to roughly quantify the number of systems that could potentially be affected by the MIEK mechanism, we changed the tidal criterion when the primary star is a giant and during mass loss to be the same as it was when the primary star was on the MS, $r_{\text {tide }}=3 R_{\mathrm{ms}, 0}$. Under this new tidal criterion, the analogue of Figure 4 is presented in Figure 5 As expected, we see a sharp decrease, from $28 \%$ to $0.011 \%$, in the fraction of triple systems that are tidally affected or collide when the primary is a giant. There are then many additional systems available after mass loss to increase the fraction of systems that are tidally affected or collide after the primary becomes a WD. The total change in the fraction is from $2.2 \%$ to $8.7 \%$. Similarly, there is a significant increase in the fraction of MIEK systems, systems that collide or become tidally affected and flip the sign of $\cos i$, from $1.9 \%$ to $6.7 \%$. For the example system considered, and for a uniform distribution in eccentricities and mutual inclination, we consider $1.9 \%$ and $6.7 \%$ to be lower and upper limits, respectively, to the fraction of all systems that undergo MIEK. 


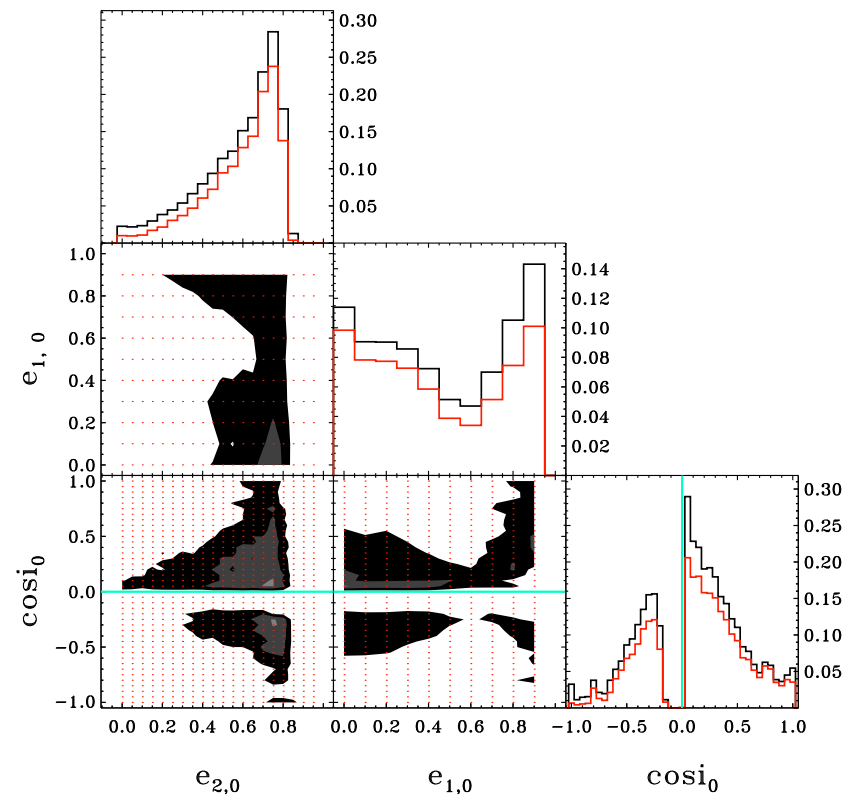

FIG. 5.- Same as Figure 4 except assuming the primary's tidal radius remains unchanged as it evolves until it becomes a WD. This is taken to be an upper bound on the fraction of systems that become tidally affected or collide after the end of mass loss. Figure discussed in $\$$

It is interesting to speculate on the outcome of triple systems that become tidally affected at each of the various points in their evolution. In what follows, we discuss the possible outcomes for tidally interacting triple systems during the MS, giant, and WD evolutionary phases of the primary in $\$ 5.1$ $\$ 5.2$ and $\$ 5.3$, respectively. We then discuss the MIEK mechanism for massive star triples during NS formation of the primary in $\$ 5.4$ Lastly, we discuss extensions of the current study in $\$ 5.5$.

\subsection{Main Sequence Phase}

We have shown in our fiducial parameter search, that systems that interact tidally while the primary is on the MS undergo both normal Kozai-Lidov oscillations and the eccentric Kozai mechanism in approximately equal proportion. Those systems that undergo normal Kozai-Lidov oscillations are analogous to the systems considered by Fabrycky \& Tremaine (2007), who calculated the long-term evolution of a distribution of triple systems using the quadrupole-order secular equations of Eggleton \& Kiseleva-Eggleton (2001). Fabrycky \& Tremaine (2007) found that the combination of normal Kozai-Lidov cycles and tidal friction produces a substantial population of close binaries, and that their calculations matched the observations of Tokovinin et al. (2006), which show that essentially all close spectroscopic binaries are actually triple systems. Given these results, we anticipate that our systems with $\cos i_{0} \sim 0$ will also tidally circularize with small $a_{1}$ and $P_{1}$, with the caveat that the MS lifetime of the primary we consider here is much shorter than for the systems considered by Fabrycky \& Tremaine (2007), and thus a fraction of our systems may not circularize before WD formation.

Importantly, though, we find that half of the systems that tidally interact or collide on the MS do so as a result of the eccentric Kozai mechanism, which, as Lithwick \& Naoz (2011), Katz et al. (2011), and Naoz et al. (2011a b) have shown cannot be captured by the quadrupole-order calculations of Fabrycky \& Tremaine (2007). What is the fate of these systems? Unfortunately, no study has yet been per- formed to investigate the eccentric Kozai mechanism with the inclusion of tidal dissipation for triple stellar systems. However, Naoz et al. (2011a) demonstrate with an octupole-order calculation that the eccentric Kozai mechanism and tidal friction can possibly explain the occurrence of retrograde hot Jupiters. They show that the extremely high eccentricities obtained by the inner binary when $\cos i$ flips signs can lead to a rapid capture of a planet into a short period retrograde orbit ("Kozai capture."). We suppose a similar mechanism operates for stellar triple systems, and that those that are tidally affected on the MS due to the eccentric Kozai mechanism rapidly dissipate energy from the inner binary and circularize with a semi-major axis of $a_{1} \approx 2 r_{\text {peri }} \sim 2 r_{\text {tide }}$. If so, then the Fabrycky \& Tremaine (2007) study may be missing of order half of the systems that become close binaries.

The action of the eccentric Kozai mechanism for MS binaries may also affect the distribution of binary eccentricities as a function of inner period as the population is circularized (Socrates et al. 2011; Dong et al. 2012).

An example observed (probable) intermediate mass triple star system that has likely already tidally circularized is MT429 in the Cygnus OB2 association, where the inner $P_{1} \simeq$ $3 \mathrm{~d} \mathrm{~B} 3 \mathrm{~V}+\mathrm{B} 6 \mathrm{~V}$ binary is orbited by a B0V tertiary at $138 \mathrm{AU}$ (Kiminki et al. 2012). An example of an even more massive system that might give rise to a NS-MS triple system as discussion in Section 5.4 is HD 150136. It consists of O3$3.5 \mathrm{~V}+05.5-6 \mathrm{~V}\left(\sim 64+40 \mathrm{M}_{\odot}\right)$ inner binary with $P_{1} \simeq 2.7 \mathrm{~d}$ and an $06.5-7 \mathrm{~V}\left(\sim 35 \mathrm{M}_{\odot}\right)$ tertiary with $P_{2} \sim 3000-5000 \mathrm{~d}$ (Mahy et al. 2012).

Finally, after the inner binary orbit has shrunk due to either normal Kozai-Lidov oscillations or the eccentric Kozai mechanism, the binary evolves like other close binaries, potentially leading to the progenitors of single degenerate (SD) supernovae (Whelan \& Iben 1973), double degenerate (DD) supernovae (Webbink 1984; Iben \& Tutukov 1984), barium stars (BA; McClure et al. 1980), and asymmetric planetary nebulae (PN) (Morris 1987).

\subsection{Giant Phase}

We find that $\sim 30 \%$ of the triple systems we consider interact tidally or collide as the primary evolves off the MS, but before mass loss, mostly as a result of normal Kozai-Lidov oscillations, which bring the pericenter of the secondary orbit inside $\sim$ AU (see right panel, Fig. 3 and Equation 17). Since the post-MS phase is short (particularly for the intermediate and high mass stars we consider here), and since the efficiency of tidal friction is uncertain, it is unclear if the system will circularize before mass loss, or whether the KozaiLidov oscillation will simply be de-tuned such that pericenter $\sim$ AU for the duration of the giant phase. The interaction of Kozai-Lidov oscillations as the primary evolves to the giant phase may also initiate mass transfer and complex secular dynamics, perhaps analogous to the system considered by Prodan \& Murray (2012). Another possibility is that this phase initiates common envelope evolution, perhaps circularizing the inner orbit at smaller semi-major axis. If the inner binary maintains significant eccentricity, or if $e_{1}$ is pumped after the giant phase and during mass loss via normal Kozai-Lidov oscillations or MIEK, such systems may form off-center or bi-polar planetary nebulae (PNe) (e.g., Soker et al. 1998; Soker \& Rappaport 2000; Witt et al. 2009). On the other hand, if the inner binary is circularized, it might also participate in the shaping of PNe (e.g., Mastrodemos \& Morris 1998, 1999). An example of some of these mechanisms in ac- 

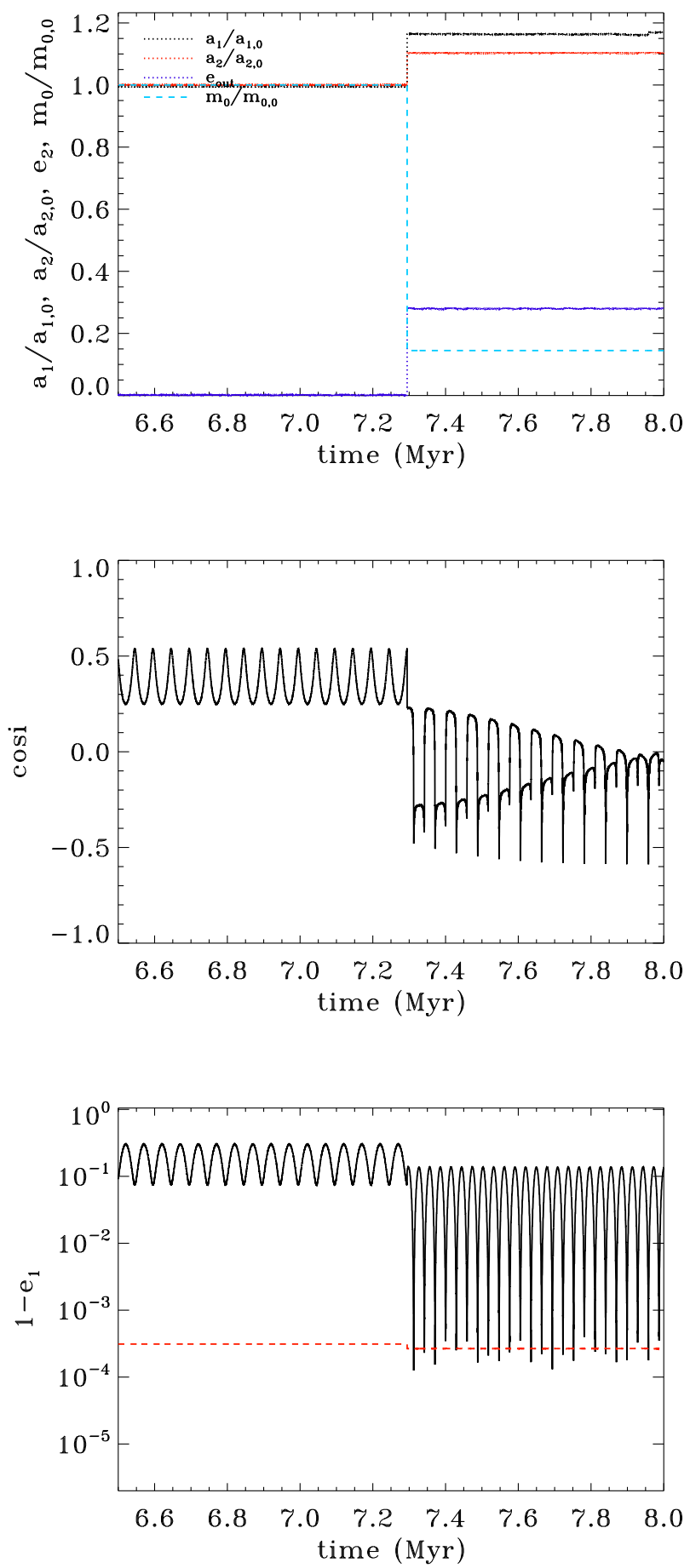

FIG. 6.- MIEK for massive stars, similar to Figure 2 but here $m_{0}=11 \mathrm{M}_{\odot}$, $m_{1}=10 \mathrm{M}_{\odot}, m_{2}=10 \mathrm{M}_{\odot}, e_{1}=0.7, e_{2}=0, \cos i=0.25, g_{1}=0^{\circ}, g_{2}=270^{\circ}$, $a_{1}=15 \mathrm{AU}$, and $a_{2}=300 \mathrm{AU}$. At $\sim 7 \mathrm{Myr}, m_{0}$ decreases to $1.4 \mathrm{M}_{\odot}, \cos i$ flips, and the system attains $1-e_{1} \sim 10^{-3}$ immediately.

tion may be ring planetary nebula SuWt 2 (Exter et al. 2010). Since a very large fraction of triple stars will be first affected by Kozai-Lidov cycles when the MS star evolves, this phase should be investigated in detail.

\subsection{After Mass Loss \& MIEK}

We find that $\sim 2 \%$ of our triple systems undergo the MIEK mechanism after WD formation (see Fig. 2). We again em- phasize that by removing all systems from our sample that were tidally affected during the giant phase, the fraction of triples that go through MIEK presented in Table 1 ("After Mass-Loss") is conservative since many of these systems may be available to go through MIEK. The reason for this is that as the primary enters its post-MS evolution and begins to interact tidally with the secondary, the orbits will be affected by processes that we do not model (e.g., Prodan \& Murray 2012). We attempt to provide an upper limit on the fraction of triple systems that could be tidally affected or collide after mass-loss due to the MIEK mechanism in Section 4.3 by assuming a small value for $r_{\text {tide }}$ during the giant phase (compare Figs. 4 and 5). This increases the fraction of triple systems that undergo MIEK from $1.9 \%$ to $6.7 \%$. A calculation of the 3-body dynamics with tides appropriate to a MS star interacting with a red giant is clearly needed to address this issue in more detail and to address the issues raised in Section 5.2 .

Systems that undergo the MIEK mechanism also have many possible outcomes. One possibility is that the extreme eccentricities obtained during a "flip" might lead to physical collisions (Baoz et al. in prep.). This may occur if, in a single orbit of the inner binary, $r_{\text {peri }}$ changes from $r_{\text {peri }}>r_{\text {tide }}$ to $r_{\text {peri }}<R_{*}$. This is possible because the angular momentum of the inner binary at such large $e_{1}$ is small. Thus, the change in angular momentum required for $r_{\text {peri }} \sim 0$ is also small. Another possible outcome for the system is a tidal Kozai capture as presented in Naoz et al. (2011a). The inner semi-major axis $a_{1}$ will strongly decrease because of tides, leaving a close MSWD binary. When the secondary subsequently evolves off the MS, the WD will either accrete or go through common envelope evolution. Both of these scenarios could lead to singleor double-degenerate (SD or DD) SNe Ia, cataclysmic variables, or other types of WD accretors such as AM CVn stars (Warner 1995), which may produce faint ".Ia" supernovae (Bildsten et al. 2007).

One interesting consequence of this evolutionary scheme for producing close WD-MS binaries is that it skips the initial common envelope evolutionary phase usually required in binary evolution to produce a close WD-MS pair. In the MIEK mechanism the close binary is produced without common envelope, and thus the WD produced should have a mass appropriate to a single star with the mass of the primary (e.g., Kalirai et al. 2008). This stands in contrast to the conventional picture for the production of WD-MS and WD-WD binaries relevant for SD and DD Ia progenitors, where the WD growth is truncated by common envelope interaction with the close secondary and the mass of the resulting WD is smaller than one would naively estimate from the single-star initialfinal mass relation (Iben \& Tutukov 1984). For SD SN Ia models, this means that less mass needs to be accreted onto the WD from the secondary, leading to a shorter delay time between production of the system and explosion. For DD SN models, if the model presented in Pakmor et al. (2012) is accurate, then the luminosity of the SN resulting from the merger of a WD-WD binary is primarily determined by the mass of the primary. Thus, all else being equal, if a WD-WD binary results from a MIEK triple system then its primary WD mass should be larger and thus result in a more luminous DD $\mathrm{SN}$ than the analogous close binary system that is produced through normal common envelope evolution.

\subsection{Massive Star Triples \& Neutron Star Formation}

The MIEK mechanism may also play an important role in triple systems after NS formation, particularly since the NS 
mass, $\simeq 1.4 \mathrm{M}_{\odot}$, is so much less than its progenitor massive star, leading to a large increase in $\epsilon_{\text {oct }}$ at the time of the associated supernova (Equation 2). However, several effects differ in the NS case versus the case of WD formation considered throughout this paper. First, NSs receive a "kick" at birth that may unbind the inner binary or the outer tertiary, depending on the masses of the constituents, their eccentricities, and their semi-major axes (Hills 1983; Kalogera 1996). Second, mass loss on the MS can affect the mass ratios as a function of time (e.g., Ekström et al. 2011). Third, even in the absence of a NS kick, the large and instantaneous mass loss in the supernova explosion from the primary provides a kick to the center of mass of the inner binary which may unbind the tertiary, and shift the mutual inclination. Even so, we expect a population of NS-MS binaries to survive with massive tertiaries and large $e_{2}$ due to the aforementioned kicks. Thus, $\epsilon_{\mathrm{oct}}$ will be large for the surviving triple systems which will cause many to exhibit the MIEK mechanism. This is a qualitatively new way to produce close NS-MS binaries.

As a test, we integrated the orbit of a system with $m_{0}=$ $11 \mathrm{M}_{\odot}, m_{1}=10 \mathrm{M}_{\odot}, m_{2}=10 \mathrm{M}_{\odot}, e_{1}=0.7, e_{2}=0, \cos i=0.25$, $g_{1}=0^{\circ}, g_{2}=270^{\circ}, a_{1}=15 \mathrm{AU}$, and $a_{2}=300 \mathrm{AU}$. At a time $\sim 7$ Myr after the start of the simulation we instantaneously decrease the mass of the primary to $1.4 \mathrm{M}_{\odot}$ and apply no kick to the NS. Figure 6 shows the resulting evolution. The system flips immediately after the supernova and evolves to an inner eccentricity of $1-e_{1}>10^{-3}$, bringing the NS to tidal contact with the secondary. Note the large increase in $e_{2}$ from $\sim 0$ to $\sim 0.3$ immediately after the $\mathrm{SN}$. This calculation is meant only to be illustrative since it does not include a NS kick. A full population study, with kicks and tides is clearly needed to assess the viability of this mechanism for forming close NSMS binaries.

A further aspect of this type of evolution is that the ratio of $a_{2} / a_{1}$ can decrease during the strong MS mass loss and the supernova explosion, which can cause the triple to become dynamically unstable and eventually disrupt, or potentially initiate collisions (the TEDI mechanism of Perets \& Kratter 2012).

\subsection{Further Studies}

In this study we have explored a single set of $m_{0}$ and $m_{1}$, however, different combinations of masses will affect the fraction of systems which become tidally affected or collide during each of the evolutionary phases. For example, if $m_{0} \approx m_{1}$ the system will have little or no time after the primary becomes a compact object before the secondary enters its giant phase. This will reduce the fraction of systems that will flip and become tidally affected or collide due to the MIEK mechanism. However, if the difference between the masses of the inner binary members is significantly greater, then a larger fraction of systems will flip before the primary goes through mass-loss. Additionally, the difference in the masses after the primary has become a compact object will be smaller, further decreasing the fraction of systems that will be affected by the MIEK mechanism. Determining the optimal balance between these two effects is beyond the scope of the current study.

In a future study we will present a more general study of mass loss in a full population of triples, quantifying the number of systems affected by Kozai-Lidov oscillations and the eccentric Kozai mechanism, an accounting of those that are unbound and those that are tidally affected or collide at each evolutionary stage, and those that undergo the MIEK mechanism.
Any study which evolves a full population of triple systems with distributions in $a_{1}$ and $a_{2}$ will have a significant fraction of systems that will be affected by the TEDI. Perets \& Kratter (2012) demonstrate that systems that start with a small $a_{2} / a_{1}$ ratio become unstable during mass-loss and evolve chaotically, leading to close encounters, collisions, and exchanges between the stellar components. Additionally, Perets \& Kratter (2012) also raise the issue of Kozai cycles and stellar evolution as important. Future studies will be needed to quantitatively evaluate the importance of both the TEDI and the MIEK mechanism.

\section{CONCLUSION}

We have presented a preliminary investigation of stellar evolution and mass loss in triple systems and demonstrated the MIEK mechanism, a novel channel through which triple systems produce compact object - MS binaries that interact tidally or collide only after the primary has evolved off the MS and become a compact object.

For a broad range of parameters, hierarchical triple star systems are unaffected by Kozai-Lidov oscillations until the primary in the central binary evolves off the MS and begins mass loss. Subsequently, the primary becomes a WD or a NS, and may then be much less massive than the other components in the ternary, enabling the "eccentric Kozai mechanism". In this near-test-particle limit, the mutual inclination between the inner and outer binary can flip signs, driving the inner binary to very high eccentricity and tidal contact or collision. In this study, we define tidal contact by an ad-hoc minimal separation between the member of the inner binary, below which tidal affects are deemed important (Section 2.2). Even distant binaries with initial semi-major axes larger then tens of AU can be strongly affected. We consider an example triple system with masses 7, 6.5 and $6 \mathrm{M}_{\odot}$ as proof -of-principle (see Fig. 2), and explore the MIEK mechanism's dependence on the initial eccentricities and inclination for this system. For a flat distribution of eccentricities and $\cos i$, we find that $13 \%, 28 \%$, and $2.2 \%$ of systems are tidally affected or collide before the primary evolves off the MS, after the primary becomes a giant, and after the primary becomes a WD, respectively.

On the MS, we find that roughly half of our systems interact tidally or collide as a result of the eccentric Kozai mechanism, and hence their dynamics would not have been captured by quadrupole-order secular calculations. In the giant phase, most of the systems are brought to tidal contact by normal Kozai-Lidov oscillations and the fact that pericenter passage occurs at semi-major axes less than $\sim$ AU even for very modest inclinations. The large fraction of such systems motivates a detailed study of the dynamics and tidal interaction of triple systems with a giant primary. Finally, the MIEK mechanism dominates the last stage, causing the WD produced by the initial primary to come to tidal contact or collide with the initial secondary which reduces $a_{1}$ without a common envelope phase. As a last application, we showed that some massive star triple systems will also undergo the MIEK mechanism after the primary's supernova explosion and the associated rapid mass loss (Figure 6).

We save a detailed study of the parameter space of triples affected by MIEK for a future paper, but here note that for a thermal distribution of eccentricities, as is commonly adopted for studies of binary stars, we expect more systems to be affected by MIEK since, in general, $\epsilon_{\mathrm{oct}}$ increases as $e_{2}$ increases (Equation 2). 
We thank Chris Kochanek, Boaz Katz, Jennifer van Saders, Joe Antognini, Krzysztof Stanek, Smadar Naoz, Yoram Lithwick, Norm Murray, and Andy Gould for discussions and encouragement. We also thank J. Fregeau for discussions and for making the code FEWBODY publicly available. This work is supported in part by an Alfred P. Sloan Foundation Fellowship and NSF grant AST-0908816. B.J.S. was supported by a Graduate Research Fellowship from the National Science Foundation.

\section{REFERENCES}

Baribault, R., \& Pineault, S. 1994, MNRAS, 271, 601

Bildsten, L., Shen, K. J., Weinberg, N. N., \& Nelemans, G. 2007, ApJ, 662 , L95

Blaes, O., Lee, M. H., \& Socrates, A. 2002, ApJ, 578, 775

Chambers, J. E., \& Migliorini, F. 1997, in Bulletin of the American Astronomical Society, Vol. 29, AAS/Division for Planetary Sciences Meeting Abstracts \#29, 1024

Dong, S., Katz, B., \& Socrates, A. 2012, arXiv:astro-ph/1201.4399

Eggleton, P. P., \& Kiseleva-Eggleton, L. 2001, ApJ, 562, 1012

Ekström, S., et al. 2011, arXiv:astro-ph/1110.5049

Fabrycky, D., \& Tremaine, S. 2007, ApJ, 669, 1298

Ford, E. B., Kozinsky, B., \& Rasio, F. A. 2000, ApJ, 535, 385

Fregeau, J. M., Cheung, P., Portegies Zwart, S. F., \& Rasio, F. A. 2004, MNRAS, 352,1

Harrington, R. S. 1968, AJ, 73, 190

Hills, J. G. 1983, ApJ, 267, 322

Holman, M., Touma, J., \& Tremaine, S. 1997, Nature, 386, 254

Iben, Jr., I., \& Tutukov, A. V. 1984, ApJS, 54, 335

Innanen, K. A., Zheng, J. Q., Mikkola, S., \& Valtonen, M. J. 1997, AJ, 113, 1915

Kalirai, J. S., Hansen, B. M. S., Kelson, D. D., Reitzel, D. B., Rich, R. M., \& Richer, H. B. 2008, ApJ, 676, 594

Kalogera, V. 1996, ApJ, 471, 352

Katz, B., \& Dong, S. 2012, arXiv:astro-ph/1211.4584

Katz, B., Dong, S., \& Malhotra, R. 2011, arXiv:astro-ph/1106.3340

Kiminki, D. C., et al. 2012, ApJ, 747, 41

Kiseleva, L. G., Eggleton, P. P., \& Mikkola, S. 1998, MNRAS, 300, 292

Kozai, Y. 1962, AJ, 67, 591

Krymolowski, Y., \& Mazeh, T. 1999, MNRAS, 304, 720

Lidov, M. L. 1962, Planet. Space Sci., 9, 719

Lithwick, Y., \& Naoz, S. 2011, arXiv:astro-ph/1106.3329

Mahy, L., Gosset, E., Sana, H., Damerdji, Y., De Becker, M., Rauw, G., \& Nitschelm, C. 2012, arXiv:astro-ph/1202.6215

Mardling, R. A., \& Aarseth, S. J. 2001, MNRAS, 321, 398

Mastrodemos, N., \& Morris, M. 1998, ApJ, 497, 303
-. 1999, ApJ, 523, 357

Mazeh, T., Goldberg, D., Duquennoy, A., \& Mayor, M. 1992, ApJ, 401, 265 Mazeh, T., \& Shaham, J. 1979, A\&A, 77, 145

McClure, R. D., Fletcher, J. M., \& Nemec, J. M. 1980, ApJ, 238, L35

Miller, M. C., \& Hamilton, D. P. 2002, ApJ, 576, 894

Morris, M. 1987, PASP, 99, 1115

Naoz, S., Farr, W. M., Lithwick, Y., Rasio, F. A., \& Teyssandier, J. 2011a, Nature, 473, 187

-. 2011b, arXiv:astro-ph/1107.2414

Pakmor, R., Kromer, M., Taubenberger, S., Sim, S. A., Roepke, F. K., \& Hillebrandt, W. 2012, arXiv:astro-ph/1201.5123

Perets, H. B., \& Fabrycky, D. C. 2009, ApJ, 697, 1048

Perets, H. B., \& Kratter, K. M. 2012, arXiv:astro-ph/1203.2914

Pinsonneault, M. H., \& Stanek, K. Z. 2006, ApJ, 639, L67

Prodan, S., \& Murray, N. 2012, ApJ, 747, 4

Raghavan, D., et al. 2010, ApJS, 190, 1

Socrates, A., Katz, B., Dong, S., \& Tremaine, S. 2011, arXiv:astro$\mathrm{ph} / 1110.1644$

Soker, N., \& Rappaport, S. 2000, ApJ, 538, 241

Soker, N., Rappaport, S., \& Harpaz, A. 1998, ApJ, 496, 842

Sterne, T. E. 1939, MNRAS, 99, 451

Thompson, T. A. 2011, ApJ, 741, 82

Tokovinin, A., Thomas, S., Sterzik, M., \& Udry, S. 2006, A\&A, 450, 681

Torres, G., Andersen, J., \& Giménez, A. 2010, A\&A Rev., 18, 67

van Saders, J. L. \& Pinsonneault, M. H. 2011, arXiv:astro-ph/1108.2273

Warner, B. 1995, Ap\&SS, 225, 249

Webbink, R. F. 1984, ApJ, 277, 355

Whelan, J., \& Iben, Jr., I. 1973, ApJ, 186, 1007

Witt, A. N., Vijh, U. P., Hobbs, L. M., Aufdenberg, J. P., Thorburn, J. A., \& York, D. G. 2009, ApJ, 693, 1946

Wu, Y., \& Murray, N. 2003, ApJ, 589, 605

Wu, Y., Murray, N. W., \& Ramsahai, J. M. 2007, ApJ, 670, 820 